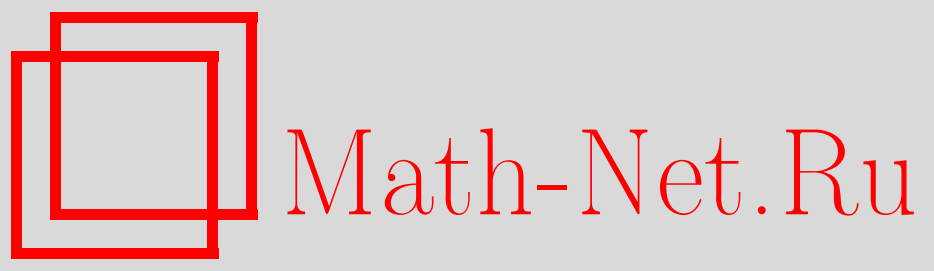

Л. Ренна, Ф. Паладини, Синусоидальные и гауссовы ударные возмущения диссипативного осцилляторного отображения, ТМФ, 2011, том 168, номер 1, 180-192

DOI: https://doi.org/10.4213/tmf6673

Использование Общероссийского математического портала Math-Net.Ru подразумевает, что вы прочитали и согласны с пользовательским соглашением http://www.mathnet.ru/rus/agreement

Параметры загрузки:

IP: 3.91 .87 .62

26 апреля 2023 г., $11: 12: 21$

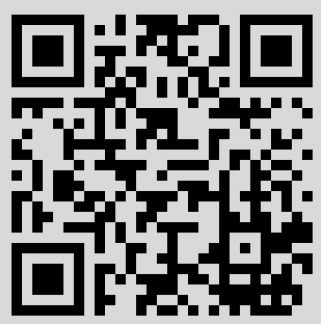




\title{
СИНУСОИДАЛЬНЫЕ И ГАУССОВЫ УДАРНЫЕ ВОЗМУЩЕНИЯ ДИССИПАТИВНОГО ОСЦИЛЛЯТОРНОГО ОТОБРАЖЕНИЯ
}

\begin{abstract}
Предлагается двумерное отображение, которое в пределе нулевой диссипации совпадает с отображением Пуанкаре для ударно-возмущенного осциллятора. Изучены основные свойства отображения под влиянием нечетных возмущений общего вида. Отображение исследовано численными методами при изменении контрольных параметров для двух различных модельных возмущений (синусоидального и гауссова), которые задают дополнительные свойства системы: в первой системе доминирующую роль играет вынужденная симметрия, а во второй - резонансная.
\end{abstract}

Ключевые слова: ударно-возмущенный осциллятор, периодические движения, хаотические отображения.

\section{1. ВВЕДЕНИЕ}

Известно, что наиболее интересным примером отображений оказываются отображения консервативного гармонического осциллятора, возмущаемого последовательностью $\delta$-образных импульсов (ударных возмущений, или киков), периодически применяемых в моменты времени $t=T, 2 T, 3 T, \ldots$ [1]. Эта система использовалась в качестве модельной при исследовании квантового хаоса в ионах в ловушках [2], [3], ионизации атомов последовательностью импульсов [4] или при изучении переноса электронов в полупроводниковых суперрешетках [5].

Добавление диссипативного слагаемого в уравнение движения гамильтоновой системы может кардинально изменить характер движения и привести к возникновению странных аттракторов. Роль, которую играют диссипативные силы в квантовых ударно-возмущенных системах, было предметом многочисленных исследований [2]-[7] в отличие от такой роли в соответствующих классических системах [8]-[10]. Поэтому в настоящей работе основное внимание уделяется пониманию свойств, привносимых диссипацией в классические ударно-возмущенные системы.

Обычно систему можно разрешить с помощью интегрирования уравнений движения по периоду $T$ с учетом того, что между двумя соседними толчками $(t \neq n T)$

*Dipartimento di Fisica, Università del Salento, Lecce, Italy.

${ }^{\dagger}$ Istituto Nazionale di Fisica Nucleare, Sezione di Lecce, Italy. E-mail: luigi.renna@le.infn.it 
решение удовлетворяет уравнению движения свободного осциллятора с затуханием, а эффект толчка сводится к добавочному мгновенному изменению скорости, зависящему от местоположения [10]. При синусиодальном возмущении и при нулевом затухании получаются хорошо известные дискретные отображения, называемые веб-отображениями [1].

Мы будем следовать другому подходу. Рассмотрим линейный осциллятор с диссипацией, находящийся под действием внешней силы $F(x)$, зависящей от координаты. Тогда получим отображение Пуанкаре при непосредственной дискретизации классических уравнений движения вынужденного гармонического осциллятора. Если затухание положить равным нулю, это отображение совпадет с отображением, сохраняющим площадь, получаемым в стандартном подходе [1]. Кроме того, это отображение проявляет все типичные свойства ударно-возмущенных систем. На самом деле дискретизация привносит дополнительную половинную степень свободы и производит эффект, эквивалентный воздействию периодической последовательности $\delta$-импульсов. Это отображение приводит также к возникновению сложного поведения и в пределе сильного подавления, в котором оно переходит в одномерное отображение, что предоставляет возможность исследования ситуации, когда стабильные гамильтоновы орбиты непрерывно связываются с одномерным отображением. Нами численно исследуется влияние двух различных видов силового члена: синусоидального возмущения, описывающего динамику заряженных частиц в поле волнового пакета, распространяющегося поперек внешнего магнитного поля, и гауссова возмущения, которое моделирует динамику отдельно взятого иона в ловушке, испытывающего нелинейные ударные взаимодействия, производимые серией гауссовых лазерных импульсов [3]. Если выполняется условие резонанса, в области хаоса параметрического пространства, где внешнее возмущение имеет характер сильного, свойства этих систем дополнительны. В первой системе доминирующую роль играет вынуждающее возмущение, и при этом наблюдается последовательность периодических состояний, сосуществующих со странным аттрактором и расположенных на одинаковых расстояниях относительно параметра вынуждающей силы; во второй системе доминирующую роль играет резонансная симметрия.

Статья имеет следующую структуру. В разделе 2 с помощью дискретизации уравнений движения диссипативного гармонического осциллятора, подвергаемого зависящему от времени внешнему воздействию общего вида, строится соответствующее отображение, а также анализируются общие свойства стабильности системы в параметрическом пространстве. Ударные возмущения синусоидального и гауссова типов исследуются соответственно в разделах 3 и 4. Наконец, обзор результатов приведен в разделе 5 .

\section{2. ОТОБРАЖЕНИЯ УДАРНО-ВОЗМУЩЕННОГО ГАРМОНИЧЕСКОГО ОСЦИЛЛЯТОРА}

Начнем с уравнения, описывающего движение линейного осциллятора, который испытывает воздействие внешней силы, зависящей от местоположения:

$$
\ddot{x}+\omega_{0}^{2} x+2 \gamma \dot{x}=F(x) .
$$

Здесь $\omega_{0}$ - угловая частота осциллятора, $\gamma$ - параметр затухания, а $F(x)$ - внешняя сила. 
Введя дискретизацию времени $t_{n}=n \Delta t$ и положив

$$
x_{n}=x(n \Delta t), \quad \frac{y_{n}}{\Delta t}=\dot{x}_{n}=\frac{x_{n}-x_{n-1}}{\Delta t}, \quad \ddot{x}_{n}=\frac{\dot{x}_{n+1}-\dot{x}_{n}}{\Delta t},
$$

получим отображение

$$
\begin{aligned}
& x_{n+1}=x_{n}+y_{n+1}, \\
& y_{n+1}=b y_{n}-Q x_{n}+f\left(x_{n}\right),
\end{aligned}
$$

где

$$
b=1-2 \gamma \Delta t, \quad Q=\left(\omega_{0} \Delta t\right)^{2}, \quad f(x)=F(x)(\Delta t)^{2} .
$$

Если $Q=0$ и $f(x)=K \sin x$ ( $K$ - параметр силы $)$, то отображение $(3)$ перейдет в хорошо известное (диссипативное) стандартное отображение, описывающее линейный ротатор, возмущенный периодически повторяющимися ударными воздействиями [11], [12].

Вводя переменные

$$
x_{n}=z_{n}, \quad y_{n}=w_{n} \sin \alpha+(1-\cos \alpha) z_{n}
$$

и полагая

$$
Q=(1+b)(1-\cos \alpha), \quad k \varphi(z)=\frac{f(z)}{\sin \alpha}, \quad \alpha=\omega \Delta t, \quad \omega=\sqrt{\omega_{0}^{2}-\gamma^{2}},
$$

можно выразить отображение (3) в терминах переменных $z, w$ следующим образом:

$$
\begin{aligned}
z_{n+1} & =\left[b w_{n}+k \varphi\left(z_{n}\right)\right] \sin \alpha+z_{n} \cos \alpha, \\
w_{n+1} & =\left[b w_{n}+k \varphi\left(z_{n}\right)\right] \cos \alpha-z_{n} \sin \alpha,
\end{aligned}
$$

где $\varphi(z)$ задает возмущение, $b$ - диссипацию, а $\alpha=2 \pi / q(q$ - целое число) - условие резонанса. Кроме того, в отличие от стандартного описания ударно-возмущенного осциллятора с диссипацией отображение (7) можно продолжить на $b=0$, где оно становится одномерным.

Независимо от вида функции $\varphi(z)$ определитель Якоби отображения (7) равен $b$. Поэтому при $b<1$ отображение относится к классу сжимающих для площади, а при $b=1$ отображение сохраняет площадь.

Рассмотрим случай внешних сил, удовлетворяющих условиям

$$
\varphi(-z)=-\varphi(z), \quad \varphi^{\prime}(0)=1
$$

В этом случае отображение имеет стационарную точку $z=w=0$. Поэтому приблизительным критерием возникновения хаоса в диссипативном случае оказывается условие нестабильности системы, расположенной в начале координат [10]. Линеаризуя уравнение (7) вблизи начала координат, получим, что фиксированная точка $(0,0)$ стабильна, когда

$$
-(1+b)(1+\cos \alpha)<k \sin \alpha<(1+b)(1-\cos \alpha),
$$

и нестабильна в противоположном случае. 
Возможные фиксированные точки с периодами 1 и 2 исследуемого отображения расположены на соответствующих прямых

$$
\begin{aligned}
& w=-z \operatorname{tg} \frac{\alpha}{2}, \\
& w=z \operatorname{ctg} \frac{\alpha}{2} .
\end{aligned}
$$

Эти точки можно определить численными методами, разрешив соответствующие нелинейные уравнения

$$
\begin{gathered}
z(1+b) \operatorname{tg} \frac{\alpha}{2}=k \varphi(z), \\
z(1+b) \operatorname{ctg} \frac{\alpha}{2}=-k \varphi(z)
\end{gathered}
$$

Условие резонанса между последовательностью толчков и частотой осциллятора $\omega$ имеет вид

$$
\alpha=\frac{2 \pi}{q},
$$

где $q$ - целое число.

Пусть $\left[k_{1}, k_{2}\right]$ - интервалы стабильности системы в начале координат при заданных значениях $b$ и $q$. При $k_{1}<k<k_{2}$ начало координат оказывается стабильной фиксированной точкой. С уменьшением $b$ ширина интервала $\left[k_{1}, k_{2}\right]$ также уменьшается; с ростом $q(>2)$ абсолютное значение отрицательной величины $k_{1}$ возрастает, в то время как $k_{2}$ монотонно убывает и стремится к нулю при $q \rightarrow \infty$. При $k>0$ система в начале координат теряет стабильность при $q>\pi / \operatorname{arctg}[k /(1+b)]$. При $k>k_{2}$ система в начале координат теряет стабильность ввиду развития бифуркации типа вилки, фиксированные точки которой задаются решениями уравнения (12), а при $k<k_{1}$ потеря стабильности обусловливается бифуркациями с удвоенными периодами, и их стабильные точки (периодические с периодом 2) можно вычислить, применив формулу (13).

При $b=1$ фазовое пространство демонстрирует поведение, характерное для гамильтоновой системы с зависимостью, периодической по времени. Такие системы характеризуются как системы, обладающие $n+1 / 2$ степенями свободы и содержащие области хаотического и квазипериодического поведения в так называемом острове. Если выполняется условие резонанса (14), то портрет фазовой плоскости обладает симметрией порядка $q$.

Нами были исследованы ударные возмущения двух различных видов - синусоидального $\varphi(z)=\sin z$ и гауссова $\varphi(z)=z e^{-z^{2}}$, которые задают системы с дополняющими свойствами: в первой из этих систем главенствующую роль играет вынужденная симметрия, а во второй - резонансная. В случае синусоидального возмущения на некоторых интервалах параметра силы $k$ наблюдается сосуществование хаотических и периодических аттракторов (аттрактор периода 1 вместе со своим быстрым каскадом удвоений периода). В случае гауссова возмущения даже при очень больших величинах параметра $k$ можно найти $q$-симметричные периодические орбиты, а хаотические состояния бифурцируют при изломах (кризисах) периодического отображения. 


\section{3. СИНУСОИДАЛЬНЫЕ УДАРНЫЕ ВОЗБУЖДДЕНИЯ}

Рассмотрим случай, когда член вынуждающей силы имеет вид

$$
\varphi(z)=\sin z
$$

При $b=1$ уравнения (7) совпадают с (бездиссипативным) веб-отображением из монографии [1]. В присутствии резонанса и для малых значений параметра $k$ формируется стохастическая сетевая структура, проявляющая свойства кристаллической $\left(q \in\left\{q_{\mathrm{c}}\right\}\right)$ или квазикристаллической $\left(q \notin\left\{q_{\mathrm{c}}\right\}\right)$ симметрии. Диссипация разрушает стохастическую сеть фазовой плоскости, и периодические аттракторы $P_{N}$ с периодами $N=n q, n=1,2, \ldots$, связанные с бифуркациями типа "седло-узел" и с удвоением периода, начинают появляться и исчезать. Если увеличивать $k$ в сторону области нестабильности состояния в начале координат, то начинают возникать хаотические области ленточного типа и странные аттракторы.

Нами при наличии резонанса были вычислены, начиная с $b=1$, последовательные представления фазовой плоскости, построенные при плавном уменьшении параметра $b$ и различных значениях параметра $k$. В ситуации общего положения при малой диссипации, введенной в систему $(0 \ll b<1)$, эллиптические клетки распадаются после (долгого хаотического) переходного периода, продолжительность которого уменьшается с уменьшением $b$, стохастическая сеть разрушается и заменяется периодическими точками. Число этих аттракторов может быть в принципе произвольно большим, если выбрать затухание достаточно малым. В наших исследованиях были найдены тысячи периодических орбит в случае параметра $b$, приближающегося к единице. Например, при $k=1.5, q=3$ и $b=0.99$, начиная с $25 \times 10^{4}$ изначальных точек внутри квадрата со стороной размера 200, с помощью метода Ньютона были найдены более $1200 P_{3}$-орбит (потомков эллиптических точек), находящихся в окружении мультипериодических состояний с периодами $3 n$ (происходящих от островов, окружающих эти точки). Такое значительное число аттракторов возникает в широком диапазоне параметрического пространства. Обычно расположение периодических точек в фазовом пространстве отражает топологию фазового пространства в точке $b=1$. В области стабильности состояния в начале координат при уменьшении $b$ мультипериодические состояния распадаются в основном при помощи бифуркаций типа "седло-узел", и число периодических состояний монотонно убывает.

Обычно с увеличением $k(>0)$ число стабильных периодических аттракторов также растет, в основном в результате бифуркаций типа "седло-узел". Этот рост может существенно прерываться возникновением изломов (кризисов). Периодические точки при этом испытывают каскады удвоений периода. Для симметричных фиксированных точек наблюдались бифуркации типа вилки, приводящие к нарушению симметрии и возникающие перед каскадным процессом удвоений периода [12]-[14]. В целом внутри области стабильности состояния в начале координат система проявляет богатое динамическое поведение, в основном состоящее в появлении и исчезновении периодических аттракторов с различными периодами (при $k \approx 2$ и $b \approx 0.95$ обнаруживаются тысячи аттракторов с максимальными периодами, превыщающими 50) при вариации параметров затухания и силы, что приводит к весьма запутанным диаграммам бифуркаций. 


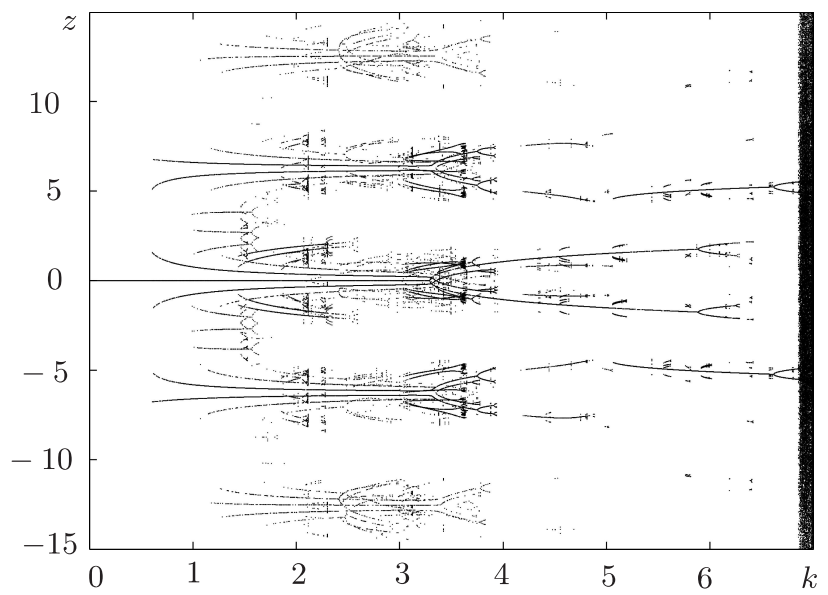

Рис. 1. Бифуркационная диаграмма для $z$ при фиксированном затухании $(b=0.9)$ и при $q=3$ при варьировании параметра силы $k(>0)$. Чтобы добиться появления такого значительного числа аттракторов (с учетом ограниченного числа начальных условий) десять начальных точек были выбраны случайным образом при каждом значении параметра $k$ в квадрате со стороной 40 и центром в начале координат. Система в начале координат остается стабильной при значениях $k \in[-1.097,3.291]$, задаваемых уравнением (9).

3.1. Бифуркационные диаграммы. Многообразие поведения систем в присутствии внешней силы (15) хорошо видно на диаграмме, показанной на рис. 1, где представлена бифуркационная структура при значениях параметров $q=3, b=0.9$. Из диаграммы отчетливо видно, что динамика в основном состоит во внезапном появлении и последующем исчезновении как периодических аттракторов с различными периодами, так и зон хаотического поведения. В области параметров, представленной на рис. 1, наблюдается множество сосуществующих аттракторов, и малая вариация параметра $k$ приводит к внезапным существенным изменениям аттрактора. Эти изменения имеют в основном характер граничных изломов (кризисов). Например, в окрестности $k \cong 2.11901$ в системе наблюдаются пара 9-компонентных хаотических аттракторов и несколько периодических аттракторов $\left(1 P_{1}, 12 P_{3}, 1 P_{4}\right.$, $\left.2 P_{9}, 2 P_{36}\right)$. С ростом $k$ хаотические области внезапно исчезают при значении параметра $k \cong 2.11902$; при этом значении $k$ в системе выживают только периодические аттракторы.

Траектории с начальными данными вблизи границы могут испытывать иррегулярное движение в течение длительного времени, прежде чем стабилизироваться на периодическом аттракторе. Это проявляется особенно отчетливо в области $k \cong 4.7-7$, где динамика характеризуется кратковременным хаосом. В общем случае области регулярного и хаотического поведения оказываются сложным образом перепутанными в зависимости от конкретных значений параметров. При $k \geqslant 7$ вблизи граничного значения $k=7$ доминирующую роль в системе играет хаотическое поведение. Тем не менее даже в этой области может наблюдаться сосуществование хаотических и периодических аттракторов внутри некоторых интервалов значений 


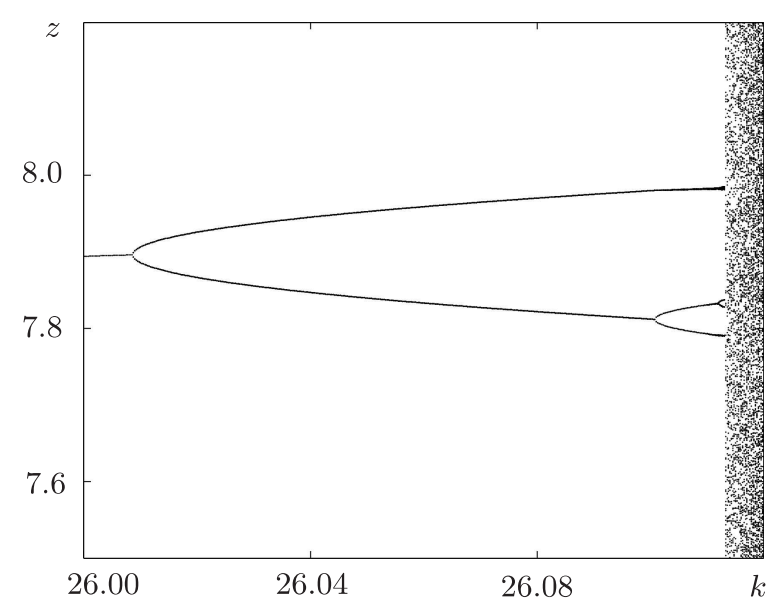

Рис. 2. Диаграмма периодических орбит $P_{1}$ и $P_{2}$, вложенных в область хаотического поведения. Параметры те же, что и на рис. 1.

параметра вынуждающей силы $k$. На самом деле орбиты $P_{1}$ и $P_{2}$ появляются в области хаоса благодаря бифуркации типа "седло-узел" и иногда благодаря бифуркации типа вилки, а затем теряют стабильность в процессе быстрого каскада удвоений периода. Это регулярное поведение проявляется вновь в областях параметра $k$, которые не удается визуализировать в бифуркационной диаграмме, так как они замаскированы сосуществующим с ними хаотическим движением. Чтобы численно найти эти орбиты на бифурукационной диаграмме, каждую траекторию, которая должна начинаться со своего собственного бассейна притяжения, не следует восстанавливать заново при каждом значении параметра $k$; ее начальными значениями нужно выбирать последние по времени точки для графика при предыдущем значении $k$. Интервалы с регулярным движением повторяются при старших значениях параметра $k$, постепенно становясь все у́же и у́же. Внутри каждой из их областей стабильности стабильные периодические орбиты с конечным периодом развиваются в соответствии со своими собственными бифуркационными последовательностями и дестабилизируются с помощью удвоения периода. Диаграмма орбит $P_{1}$ и $P_{2}$, стабильных относительно амплитуды возмущения $k$, показана на рис. 2.

Размеры бассейнов притяжения этих периодических аттракторов сравнительно малы по сравнению с размерами бассейнов притяжения хаотических аттракторов (примеры бассейна притяжения фиксированных точек показаны на рис. 3), и эти бассейны быстро уменьшаются с ростом $k$, так что они вряд ли могут возникать из обычной бифуркационной диаграммы. Однако эти траектории можно детально проанализировать, поскольку они получаются из периодичности возмущающего члена.

3.2. Фиксированные точки в области хаоса. Нетрудно найти $P_{1}$-орбиты в бифуркационной диаграмме. Пусть $z^{*}$ и $w^{*}$ представляют собой координаты фиксированной точки общего положения. Одно из свойсти якобиана уравнения (7) состоит в том, что он зависит только от $z^{*}$. Из условия стабильности следует уравнение

$$
-(1+b)<k \varphi^{\prime}\left(z^{*}\right) \sin \alpha+(1+b) \cos \alpha<(1+b) .
$$




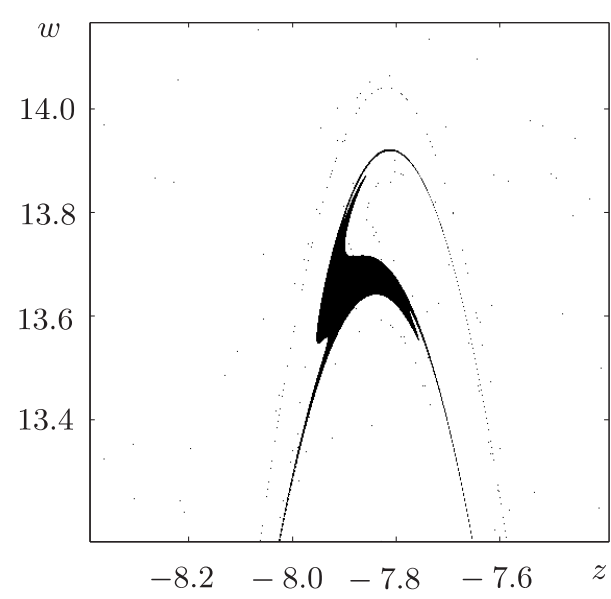

a

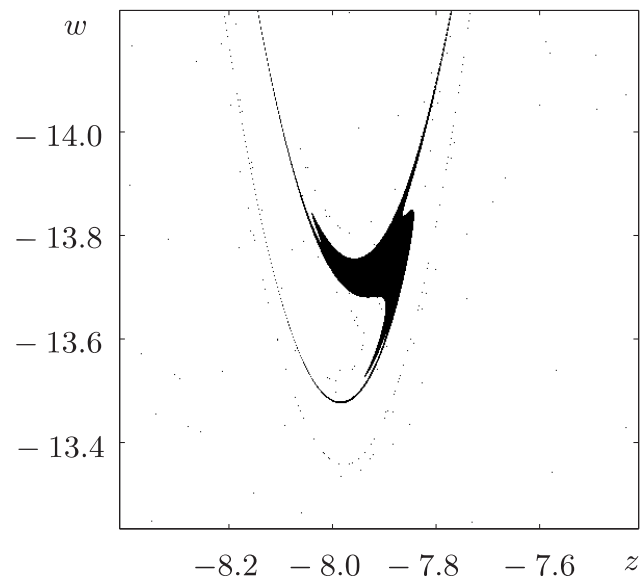

б

Рис. 3. Бассейны притяжения $P_{1}$-аттракторов при $k=25.85$ (другие параметры те же, что и на предыдущих рисунках). Отметим примечательную симметрию бассейнов относительно пространственных отражений.

Получив $k$ из уравнения (12), получим при $q>2$ условие

$$
-\operatorname{ctg}^{2} \frac{\alpha}{2}<z^{*} \frac{\varphi^{\prime}\left(z^{*}\right)}{\varphi\left(z^{*}\right)}<1
$$

Если $\varphi(z)$ задается формулой (15), то из этого условия следует, что

$$
-\operatorname{ctg}^{2} \frac{\alpha}{2}<z^{*} \operatorname{ctg} z^{*}<1 .
$$

Очевидно, что условие (18) явно выполнено для

$$
\begin{array}{llrl}
z_{m}^{*} & = \pm \frac{\pi}{2} \pm 2 m \pi, & & k>0, \\
z_{m}^{*} & = \pm \frac{\pi}{2} \pm(2 m+1) \pi, & & k<0 .
\end{array}
$$

Применив уравнение (12), получим, что эти точки располагаются на кривой

$$
k=\frac{z}{\sin z}(1+b) \operatorname{tg} \frac{\alpha}{2},
$$

имеющей производную

$$
\left.\frac{d k}{d z}\right|_{z=z^{*}}= \pm(1+b) \operatorname{tg} \frac{\alpha}{2} .
$$

Значения $w^{*}$ на фазовой плоскости, отвечающие $z^{*}$, задаются уравнением $(10)$, так что они располагаются на кривой, имеющей производную

$$
\left.\frac{d k}{d w}\right|_{w=w^{*}}=\mp(1+b),
$$




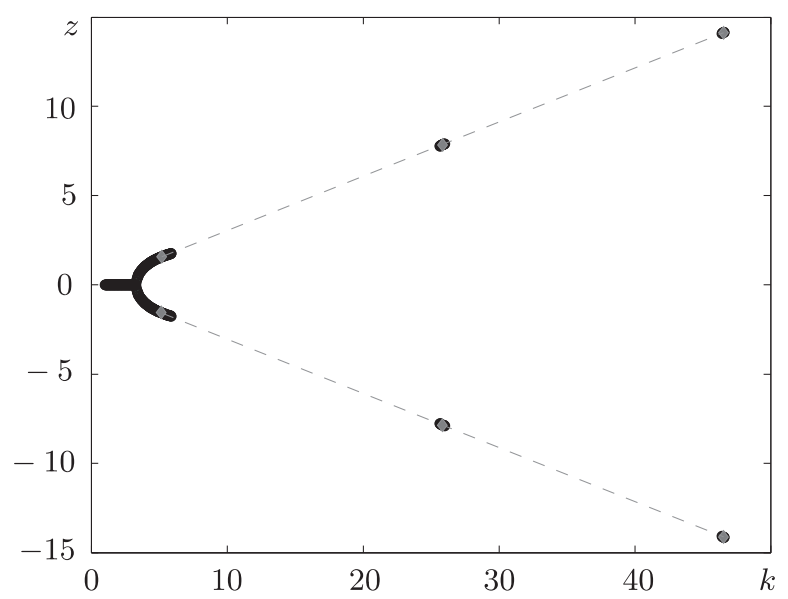

Рис. 4. Фиксированные точки $P_{1}$, погруженные в область хаоса. Точками обозначены фиксированные значения $z_{m}^{*}$, предсказываемые уравнением (19). Выбраны следующие значения параметров: $b=0.9, q=3$.

которая не зависит от $q$. Нетрудно увидеть, что значения $z^{*}$ располагаются на равном удалении друг от друга в $k$ :

$$
\Delta k_{m}=2 \pi(1+b) \operatorname{tg} \frac{\alpha}{2} .
$$

Местоположения фиксированных точек $z^{*}$ в бифуркационной диаграмме можно получить численно с применением метода Ньютона или просто с помощью уравнений (18), (20). С помощью обоих этих методов получаются графики, весьма напоминающие представленные на рис. 4, где изображены периодические точки бифуркационной диаграммы, погруженной в область хаоса. Точками обозначены значения, предсказываемые уравнением (19). Области регулярного поведения бифуркационного графа увеличиваются в размере с ростом $q$. Это не так уж и неожиданно с учетом того, что с ростом $q$ также увеличивается ширина интервала (18), а абсолютное значение производной (21) уменьшается.

Эти свойства имеют весьма общий характер, так как они типичны для осциллятора, подвергаемого воздействию периодической внешней силы. При $\varphi(z)=$ $\cos z$ из неравенства (17) получим, что в областях значений $z$, сконцентрированных вокруг точек $z= \pm m \pi, m=0,1,2, \ldots$, имеются фиксированные точки, а из уравнения (12) получим, что соответствующие значения параметра имеют вид $k= \pm m \pi(1+b) \operatorname{tg}(\alpha / 2)$ при четных $m$ и $k=\mp m \pi(1+b) \operatorname{tg}(\alpha / 2)$ - при нечетных. Интересно отметить, что функция $\varphi(z)=\cos z$ не обладает свойством (8). Таким образом, в случае периодического возмущающего воздействия можно найти фиксированные точки при каждом, в том числе сколь угодно большом, значении параметра $k$. Это является следствием противодействия между частотами осциллятора и вынуждающей силы. 


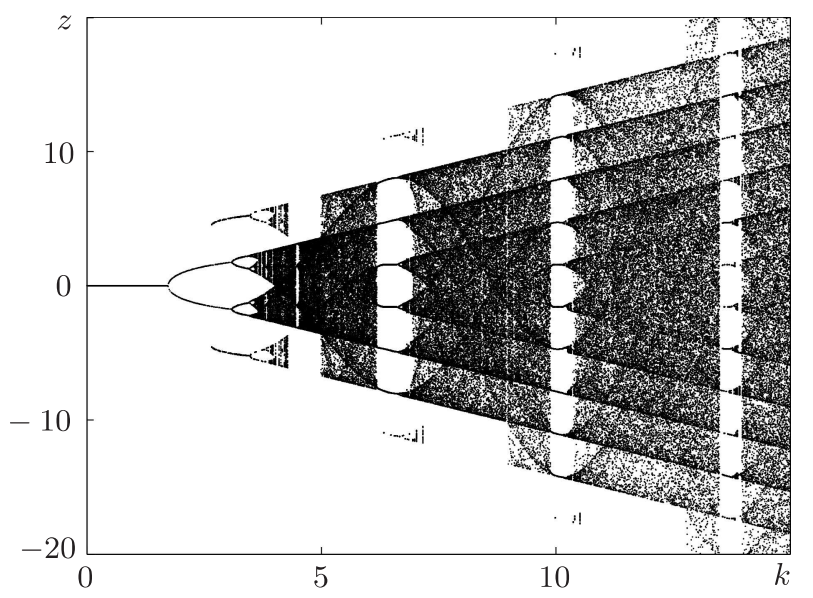

Рис. 5. Та же диаграмма, что и на рис. 1 , но при значении параметра $b=0$.

3.3. Предел сильной абсорбции $(b=0)$. Отображение $(7)$ можно продолжить на предельный случай очень сильного подавления $b=0$, в котором оно перейдет в одномерное отображение (как это имеет место в случае стандартного диссипативного отображения [11], [12]).

Относительная бифуркационная диаграмма, представленная на рис. 5, имеет вид, типичный для одномерных отображений: с хаосом, каскадами удвоений периодов, окнами периодичности, областями хаотического поведения, изломами (кризисами) и т. д. Характерными для диаграммы оказываются внутренние изломы странных аттракторов. Например, видно, что вблизи $k \approx 4.3$ на диаграмме возникает излом двухкомпонентного аттрактора, сосуществующего с однокомпонентным аттрактором. Этот второй хаотический аттрактор сталкивается с седловым аттрактором периода 2 примерно при $k \approx 5.0$, скачком меняя при этом размер, испытывая внутренний излом. Тем самым из двух аттракторов, существующих при $k \approx 4.25$, первый исчезает, испытывая граничный излом, а затем появляется вновь при $k \approx 5$, чтобы образовать вместе со вторым единый аттрактор, возникновение которого обусловлено внутренним изломом. Также для бифуркационной диаграммы характерно наличие последовательности окон периодичности, размер которых уменьшается с ростом $k$. На рис. 5 видно, что, начиная примерно с $k=4$, диаграмма состоит из последовательности хаотических областей, каждая из которых прерывается внезапным появлением стабильной периодической орбиты с конечным периодом и последующей бифуркацией. Окна периодичности повторяются при почти равноудаленных значениях параметра $k$. Вне каждого окна между двумя аттракторами развивается излом, и это явление повторяется с той же частотой, с которой появляются окна, следующие за этими изломами. При больших значениях параметра $k$ окна периодичности заполняются областями хаотического поведения, и такая структура исчезает.

Фиксированные точки, сходные с полученными в предыдущем разделе $(b \neq 0)$, продолжают сосуществовать в бифуркационных диаграммах с тем же расположением, что представлено на рис. 4, но они отнесены по параметру $k$ чуть больше, 
чем на $1 / 2$. Их не видно, так как они погружены в сосуществующие хаотические аттракторы.

\section{4. ГАУССОВЫ ИМПУЛЬСЫ}

Классическую динамику отдельного иона в ловушке, который испытывает нелинейные ударные воздействия, производимые последовательностью гауссовых лазерных импульсов, можно моделировать с помощью функции

$$
\varphi(z)=z e^{-z^{2}}
$$

Чтобы найти фиксированные точки, произведем подстановку

$$
z \frac{\varphi^{\prime}(z)}{\varphi(z)}=1-2 z^{2}
$$

в уравнении (17), что дает условие

$$
-\operatorname{ctg}^{2} \frac{\alpha}{2}<1-2 z^{* 2}<1
$$

которому удовлетворяет функция

$$
z^{* 2}<\frac{1}{2} \operatorname{cosec}^{2} \frac{\alpha}{2}
$$

Из уравнения (12) получим, что соответствующие величины параметра $k$ при $z^{*} \neq 0$ имеют вид

$$
k^{*}=(1+b) e^{z^{* 2}} \operatorname{tg} \frac{\alpha}{2} .
$$

Если $k$ удовлетворяет условию (9), то начало координат оказывается стабильной фиксированной точкой, испытывающей удвоение периода при $k=-(1+b) \operatorname{ctg}(\alpha / 2)$ и бифуркацию типа вилки при $k=(1+b) \operatorname{tg}(\alpha / 2)$. Эти последние фиксированные точки остаются стабильными вплоть до значения параметра $k=(1+b) \operatorname{tg}(\alpha / 2) e^{\operatorname{cosec}^{2}(\alpha / 2) / 2}$, при котором каскад удвоений периодов приводит к хаосу. На рис. 6 изображен график бифуркационной диаграммы динамической переменной $z$ относительно параметра внешней силы $k$. На диаграмме показана последовательность перекрывающихся периодических орбит, так что реализуется четкое разделение между периодическим и хаотическим поведением. Поскольку вынуждающая сила не совпадает с собственной частотой, динамика в основном определяется частотой гармонического осциллятора. На рис. 7 показаны два странных аттрактора, характерных для системы. При уменьшении $b$ аттракторы коллапсируют на прямую, как и в случае одномерных отображений. Странный аттрактор при $b=0$ окрашен в серый цвет на рис. 7б).

\section{5. ЗАКЛЮЧИТЕЛЬНЫЕ ЗАМЕЧАНИЯ}

В работе было предложено новое отображение, происходящее из одномерного гармонического осциллятора с затуханием, испытывающего воздействие внешнего возмущения, зависящего от местоположения. В пределе нулевой диссипации это 


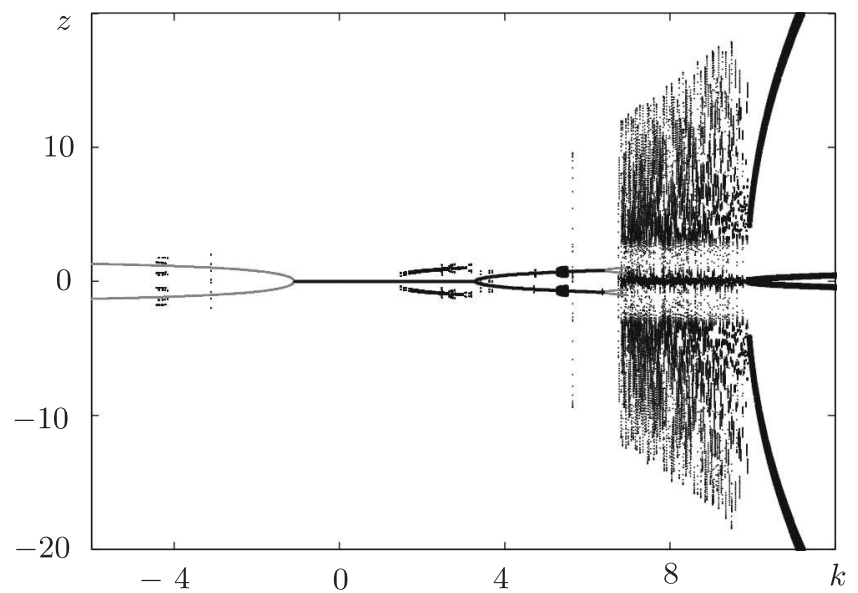

Рис. 6. Бифуркационная диаграмма величины $z$ в зависимости от $k$ в случае, когда возмущение моделируется уравнением (24). Параметры принимают те же значения, что и на рис. 1 . На диаграмме показаны точки пересечения $P_{1}$ (черные тонкие линии), $P_{2}$ (серые линии) и $P_{3}$ (черные жирные линии). При $k>10$ выживают только два состояния $P_{3}$ за исключением очень высоких значений параметра $k\left(k \approx 10^{25}\right)$.

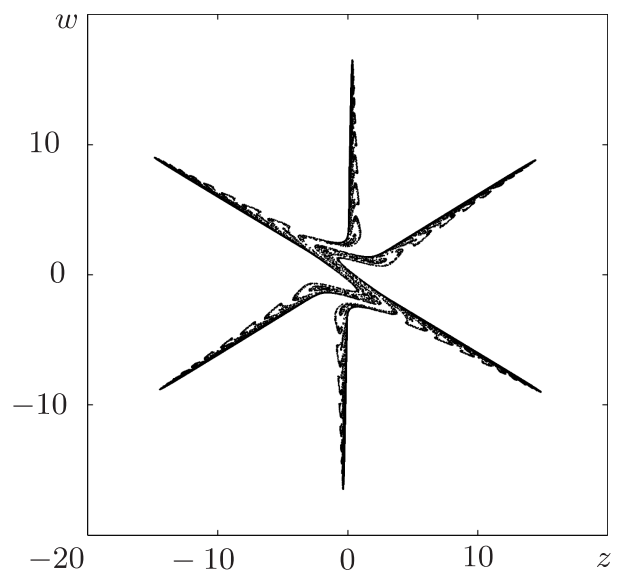

a

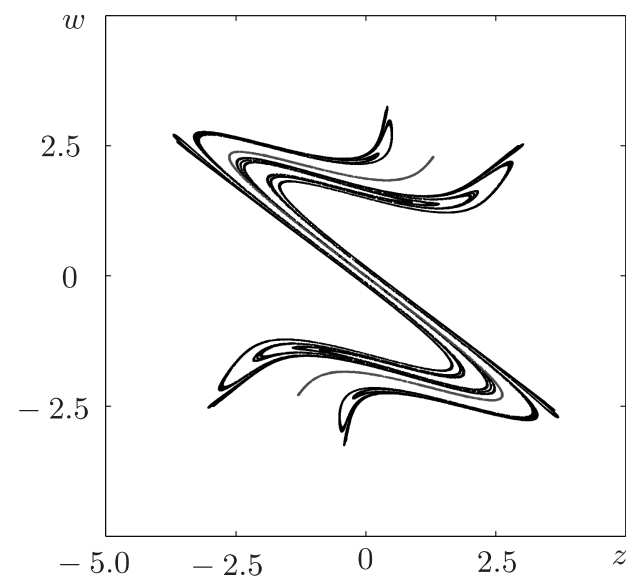

6

Рис. 7. Хаотические аттракторы при $k=8, q=3: b=0.9$ (а); $b=0.5-$ черная линия и $b=0$ - серая линия (б).

отображение совпадает с отображением Пуанкаре ударно-возмущаемого осциллятора, испытывающего воздействие последовательности периодических толчков (так называемое веб-отображение).

Без потери общности были проанализированы, в частности, свойства дискретно возмущаемого гармонического осциллятора при резонансном значении $q=3$, под- 
вергаемого синусоидальному и гауссову возмущениям, что моделирует различные физические системы. Эти возмущения приводят к дополняющим друг друга свойствам: в первой системе главенствующую роль играет вынужденная симметрия, а во второй - резонансная симметрия. Примерно одни и те же динамические характеристики наблюдаются в системах с различными значениями параметра $q$ (кроме $q$-симметрии). При $b=0$ отображение не теряет своей зна́чимости, как в случае стандартного подхода к ударно-возбужденному осциллятору, но сжимается до прямой, становясь при этом одномерным.

Отображение представляет собой богатую и разнообразную динамическую систему, демонстрирующую очень сложное поведение в различных областях параметрической плоскости. Анализ динамики в параметрическом пространстве показывает сосуществование множества периодических и хаотических аттракторов, каскадов удвоений периодов с переходом в хаос, изломов (кризисов), кратковременных переходов в хаос, странных аттракторов и т.д. также и при малых значениях параметра $b$. Нами было найдено множество сосуществующих аттракторов (тысячи периодических состояний), чьи бассейны притяжения переплетены очень сложным образом. Большинство периодических аттракторов возникает в результате бифуркаций типа "седло-узел".

Исследование отображения в присутствии возмущений различных видов может пролить свет на роль диссипации в квантовых системах, где аналогичные задачи подходящим образом моделируются с помощью квантовых аналогов диссипативных ударно-возмущенных гармонических осцилляторов.

\section{Список литературы}

[1] G. M. Zaslavsky, Physics of Chaos in Hamiltonian Systems, London, Imperial College Press, 1998.

[2] S. A. Gardiner, J. I. Cirac, P. Zoller, Phys. Rev. Lett., 79:24 (1997), 4790-4793, arXiv: quant-ph/9707058; Erratum: 80:13 (1998), 2968-2968.

[3] A. J. Scott, C. A. Holmes, G. J. Milburn, Phys. Rev. A, 61:1 (2000), 013401, 7 pp., arXiv: chao-dyn/9903012.

[4] S. Tanabe, S. Watanabe, N. Sato, M. Matsuzawa, S. Yoshida, C. Reinhold, J. Burgdörfer, Phys. Rev. A, 63:5 (2001), 052721, 11 pp.

[5] T. M. Fromhold, A. A. Krokhin, C. R. Tench, S. Bujkiewicz, P. B. Wilkinson, F. W. Sheard, L. Eaves, Phys. Rev. Lett., 87:4 (2001), 046803, 4 pp.

[6] A. Iomin, G. M. Zaslavsky, Phys. Rev. E, 67:2 (2003), 027203, 4 pp.

[7] A. R. R. Carvalho, R. L. de Matos Filho, L. Davidovich, Phys. Rev. E, 70:2 (2004), 026211, 15 pp., arXiv: quant-ph/0405009.

[8] L. Renna, Phys. Rev. E, 64:4 (2001), 046213, 7 pp.

[9] R. F. Mudde, S. G. Jansz, Physica D, 179:1-2 (2003), 1-17.

[10] A. A. Vasil'ev, G. M. Zaslavsky, M. Ya. Natenzon, A. I. Neüshtadt, B. A. Petrovichev, R. Z. Sagdeev, A. A. Chernikov, ЖЭЭФ, 94:10 (1988), 170-187.

[11] G. M. Zaslavsky, Phys. Lett. A, 69:3 (1978), 145-147.

[12] G. Schmidt, B. W. Wang, Phys. Rev. A, 32:5 (1985), 2994-2999.

[13] J. W. Swift, K. Wiesenfeld, Phys. Rev. Lett., 52:9 (1984), 705-708.

[14] U. Feudel, C. Grebogi, B. R. Hunt, J. A. Yorke, Phys. Rev. E, 54:1 (1996), 71-81. 